\title{
Comparison of Two Different Methods for Coherent Averaging in Online ECG Analysis
}

\author{
K Egorouchkina ${ }^{1,2}$, M Braecklein ${ }^{2}$, L Pang $^{2}$, I Tchoudovski $^{2}$, \\ W Kellermann ${ }^{1}$, A Bolz ${ }^{2}$ \\ ${ }^{1}$ Friedrich - Alexander - Universität Erlangen-Nürnberg, Erlangen, Germany \\ ${ }^{2}$ Corscience $\mathrm{GmbH} \&$ Co KG, Erlangen, Germany
}

\begin{abstract}
The coherent averaging is an often used method in ECG systems for noise removing. Most algorithms are developed for the offline data analysis either with automatic or with manual pattern determination for cycles averaging [1-3]. These algorithms can't be used for a wearable long time ECG analysis system [4], which processes the biosignal continuously in real time, because the continuous analysis needs a lot of computational power, which can be provided only by PCs. Therefore, an online averaging algorithm was developed for the ECG signal filtering for wearable long time analysis systems. This article presents an effective algorithm for online ECG complex averaging. Also a comparison of two methods for cycle allocation is offered.
\end{abstract}

\section{Introduction}

The electrocardiogram (ECG) provides almost all information about electrical activity of the heart. The ECG signal is a sequence of ECG-cycles. Normally each cycle consists of a succession of $\mathrm{P}$ wave, QRS complex, $\mathrm{T}$ wave, and sometimes $\mathrm{U}$ wave. Each of these waves shows an electrical passing in the heart. While the $\mathrm{P}$ wave corresponds to the depolarisation of the atria, the QRS complex shows the ventricular depolarisation and the $\mathrm{T}$ wave is the ventricular repolarisation of the human heart. Automatic ECG wave delineation should be very effective for a reliable ECG parameter determination. The search of the fiducial point should be performed on ideally not pre-filtered ECG sequences in order not to distort the diagnostic information. An effective ECG wave recognition is difficult because of different noises. The noises can be filtered with digital filters. In this case the ECG signal cans loss diagnostic information, because most of the filters change the signal significantly in time and frequency domain. On the other hand the noise can be removed by averaging over several ECG cycles. This is shown in figure 1 . Averaging $N$ heartbeats will improve the signal-to-noise ratio by a factor of $\sqrt{N}$ [3].

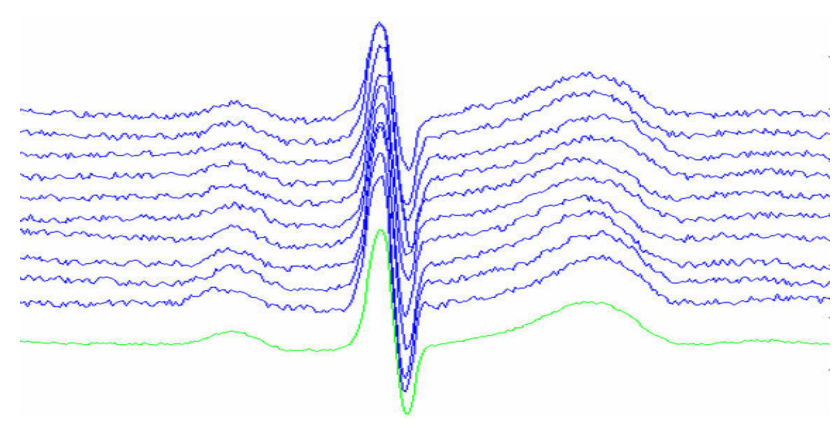

Figure 1. An example of coherent averaging with $\mathrm{R}$ peak trigger point.

An averaged complex has a higher signal-to-noise ratio, more reliable fiducial points and less isoline drift than the original complex. High ECG cycle quality for the online ECG parameter analysis can be achieved with the selective time-coherent averaging in real time.

Recent developments of microcontrollers allow the continuous monitoring of high risk patients with wearable devices. There a not too complex algorithm is needed to save computational power and energy.

\section{Methods}

\subsection{Algorithm}

\subsubsection{Algorithm concept}

The following steps are necessary for the real time selective averaging algorithm. First ten seconds are used only for the calculation of the mean RR interval and for the parameter adaptation. All the following intervals of ten seconds are used for cycle allocation and for recalculation of the mean RR intervals, as shown in figure 2. Averaging and analysis of ECG complexes are executed on raw data without digital filtering. The algorithm starts the complexes allocation procedure after QRS complex detection and trigger point determination. The complex allocation is executed on a basis of given 
criteria. At the end of the ten second interval an averaged beat is calculated and the average cycle length is recalculated depending on a new mean RR interval. Then the averaging procedure is restarted. In the following each point is described in detail.

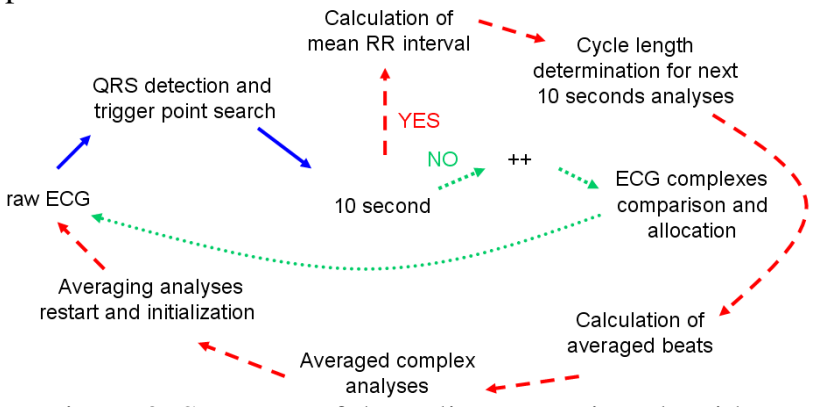

Figure 2. Structure of the online averaging algorithm.

\subsubsection{QRS complex detection and trigger point search}

To get a representative cycle for a patient, the ECG complexes should be time-coherent averaged relative to a trigger point. $\mathrm{R}$ peak (or $\mathrm{Q}$ peak, if the $\mathrm{R}$ peak amplitude is much smaller than the $\mathrm{Q}$ peak amplitude) gives the best possibility for the cycle synchronisation, because this wave is the most noticeable in an ECG complex. The trigger point is determinated after QRS complex detection. It is the max or min amplitude in an assigned area of detected QRS complex. The trigger point type (negative or positive peak) is defined once time per interval analysis. The used QRS detection algorithm is described in [5]. The QRS detection is verified using standard databases from Physionet. Sensitivity and positive predictivity are above $99 \%$ for QT databases [6].

\subsubsection{ECG cycle length determination}

The ECG cycles for averaging should include a whole ECG complex from the start of atria activation ( $P$ wave onset) to the end of the ventricular repolarisation ( $\mathrm{T}$ or $\mathrm{U}$ wave offset). The cycle length should be constant for all complexes in ten seconds analysis interval and should be determined before complex allocation. The ECG cycle duration is calculated on the basis of a mean RR interval value of the preceding ten seconds. The cycle length is generated as sum of time intervals before and after $\mathrm{R}$ peak. The interval before and after $\mathrm{R}$ peak are defined as $C_{1} \cdot \overline{R R}_{10 s}$ and $C_{2} \cdot \overline{R R}_{10 s}$ respectively. There $C_{1}=2 / 3$ and $C_{2}=9 / 10$ are empirical coefficients. $\overline{R R}_{10 s}$ is the mean RR interval value of the preceding ten seconds. In this way the defined ECG cycle length includes the whole ECG complex without information loss.

Furthermore the mean RR interval adapts dynamically to patient rhythm, because it is calculated each ten seconds

\subsubsection{Selective ECG cycles allocation}

The selective ECG cycle allocating is performed in the following way. The first ECG complex is always ordered to the first class. The second and all the next beats are compared with the template from the existing classes starting with the first one. In case a beat candidate and the pattern in the group are almost the same, this beat is added to this class. The beat candidate creates a new class, if there isn't a similar class template to the beat candidate.

The group template is always the first saved beat in the class. Otherwise, there may be just small differences between single members of the class but a big difference between the first and the last cycle. On the other hand, the averaged beat over all saved complexes in a group as group template needs a long computation time.

Altogether six classes are possible. The sixth class contains all rest beats, which weren't ordered to available classes. This cycle allocation method is adaptive for each patient.

\subsubsection{ECG cycle averaging}

The ECG recording is allocated in groups and each group is averaged every ten seconds according to:

$$
A v(t)=\frac{1}{N} \sum_{N} Z_{n}(t)
$$

where $A v$ - is an averaged beat in a group, $Z n$-is a single cycle of the group, $N$ - is the number of cycles in the group, $t=1 \ldots T, T-$ is the cycles length.

A ten second interval is chosen for averaging because this is the standard time for short time ECG analysis. The averaged cycle of the largest class present the typical ECG complex or representative beat for this patient at a given moment. The analysis is restarted every ten seconds after getting representative complex.

\subsection{ECG complex comparison}

The most difficult part in the described process is to compare two ECG cycles correctly. The degree to which candidate and template cycles are related must be estimated for an effective ECG complex allocation. Two methods for ECG complexe allocation are developed and compared (figure 3 ).

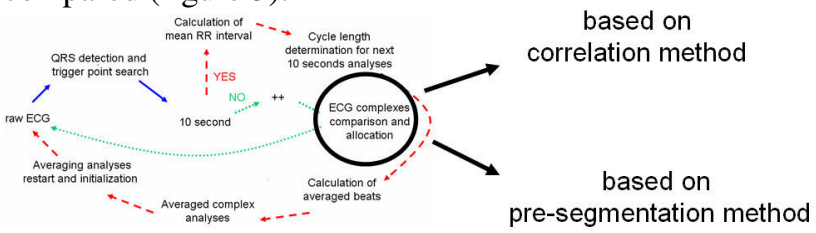

Figure 3. Methods for the beat allocation 


\subsubsection{Method based on correlation}

The mathematical approach to estimate the similarity of the signals is the correlation. The first method is bases on the correlation of whole candidate complex with the template in the group. The procedure of the allocation method based on correlation works like:

(1) Detect a QRS complex and determine the trigger point as described in 2.1.2.

(2) Store the ECG cycle $X=[x(1), x(2), \ldots, x(T)]$ with the length determined in 2.1.3

(3) Calculate the correlation coefficient $r_{x y}$ between candidate complex $X$ and group template $Y$ :

$$
r_{x y}=\frac{T \sum x(i) y(i)-\sum x(i) \sum y(i)}{\sqrt{T \sum x(i)^{2}-\left(\sum x(i)\right)^{2}} \sqrt{T \sum y(i)^{2}-\left(\sum y(i)\right)^{2}}}
$$

where $i=1 \ldots T, T-$ is the ECG cycle length.

(4) Add the stored ECG cycle candidate to the group, if the relationship between template in a group and complex candidate exceeds a given threshold magnitude. Different thresholds $(0.90,0.95$ and 0.97$)$ of correlation coefficient are analysed to find the best relation degree in an averaging group.

(5) Each ten seconds calculate the averaged beat as in 2.1.5.

\subsubsection{Method based on pre-segmentation}

The correlation method needs a lot of calculation. Therefore, a second method for ECG complex allocation is developed. The second method allocates the ECG segments by an ECG complex character vector. The character vector contains both ECG complex amplitudes $(P, R, S, T)$ and intervals $(P R, R T)$ as shown in figure 4.

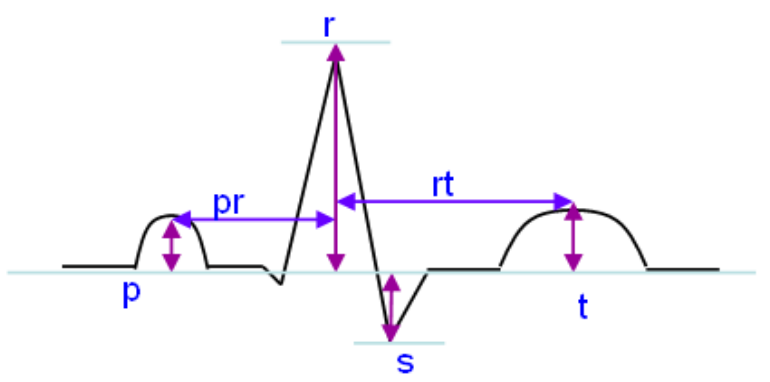

Vcycle $=[p, r, s, t, p r, r t]$

Figure 4. ECG complex character vector

These chosen parameters are the most reliable by ECG complex delineation. The ECG character vector is obtained for each complex in real time with a segmentation algorithm (described below).

The procedure of the allocation method based on pre- segmentation performs the following steps:

(1) Detect a QRS complex and determine the trigger point as described in 2.1.2.

(2) Store the ECG cycle $X=[x(1), x(2), \ldots, x(T)]$ with the length determined in 2.1.3

(3) For each ECG cycle $X=[x(1), x(2), \ldots x(M)]$, find the ECG vector Vcycle $=[p, r, s, t, p r, r t]$ ( see 2.2.3.)

(4) Normalize Vcycle :

(5) Calculate the difference between candidate cycle and template in a group:

$\operatorname{Diff}(j)=a b s\{\operatorname{Vcandidate}(j)-\operatorname{Vtemplate}(j)\}$

(6) Add the stored ECG cycle candidate to the group, if the difference doesn't cross given thresholds. The thresholds were determinate empirical.

(7) Each ten seconds calculate the averaged beat as described in 2.1.5.

\subsubsection{ECG segmentation algorithm}

The pre-segmentation algorithm starts the ECG wave delineation after QRS complex detection. The R and S peak are determined as max and min amplitude in the QRS area. This area is calculated on basis of the RR interval. The search window for the peaks is defined from the QRS position whose limits are RwindowBeforeQRS and RwindowAfterQRS [7].

$\begin{aligned} & \text { (RwindowBeforeQRS, } \\ & \text { RwindowAfterQRS) }\end{aligned}=\left\{\begin{array}{l}140 \mathrm{~ms}, \text { if } \mathrm{RRmean}>700 \mathrm{~ms} \\ 100 \mathrm{~ms}, \text { if } \mathrm{RRmean} \leq 700 \mathrm{~ms}\end{array}\right.$

$\mathrm{P}$ and $\mathrm{T}$ wave are searched before and after QRS complex respectively in a given time duration. The determination of the time area for the $\mathrm{P}$ and $\mathrm{T}$ wave search is executed dynamically depending on the RR interval.

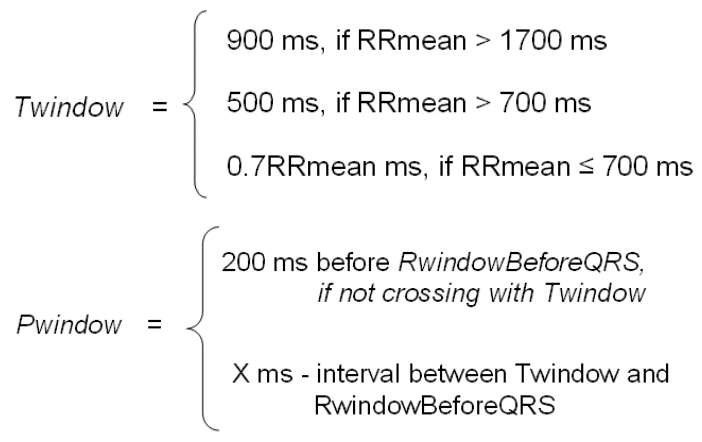

The mean RR interval is used to avoid significant changes in the $R R$ interval. The mean $R R$ value is calculated on the basis of the three last intervals.

The determination of the wave amplitude is released by 
analysis of ECG signal together with the derivative of ECG signal. The wave peak is found if the max or min amplitude in the given ECG area corresponds to a derivative zero-crossing.

The developed algorithm is tested on QT-database from Physionet [6]. The sensitivity for $\mathrm{P}$ and $\mathrm{T}$ waves amplitudes are around $92 \%$ and $95 \%$ respectively.

\section{Results}

The CSE [8] and QT [6] databases are used for algorithm verification and method comparison. The test is conducted with only the first two minutes of the QT database and with the repeated II lead of the CSE databases.

\subsection{Evaluation method}

The validation and comparison are executed in three steps. The first one is the number of groups per ten seconds interval.

The second step is evaluation of the quality of the averaged group. The calculated averaged beat for the largest group is compared with all beats from this group by a mean square error. Suppose $A v$ is an averaged beat in a group and $Z n$ is a complex from this group, then the error can be calculated like:

$$
\operatorname{Err}_{n}=\frac{1}{T} \sum_{T}\left(Z_{n}-A v\right)^{2}
$$

If the averaged beat isn't a member in the group then the mean square error has a big deviation in comparison with other errors in this group. In that way the number of the incorrect beats in a group can be calculated.

The third step is the evaluation of the quality of the method. In this case the averaged mean square error for the group is computed. The averaging is executed over all mean square errors in this group:

$$
\text { Errav }=\frac{1}{N} \sum_{N} \operatorname{Err}_{n}
$$

There $N$ is the number of cycles in a group.

The best result is given by the method with minimal number of groups and a minimal mean square error in the largest group.

\subsection{Result of comparison}

The best performance is achieved with the method based on the correlation coefficient 0.95 . The correlation method with correlation coefficient under 0.92 puts together normal complexes and extrasystoles into the same group. In this group one gets the biggest mean square error. Many groups with similar complexes provide correlation above 0.96 . In this case the number of the different groups is the largest.
The method based on pre-segmentation is comparable with the correlation method with a coefficient of 0.95 . The correlation method shows a little better result.

However, the pre-segmentation method has much smaller cost of computation.

\section{Discussion and conclusions}

For coherent averaging in online ECG analysis two effective algorithms are developed and compared.

The algorithm based on the correlation coefficient gives the best performance and can be used for online averaging on a PC.

In online ECG analysis on a microprocessor for wearable devices the method based on pre-segmentation is more suitable, because the computation costs are much smaller for this method and the result of both methods are nearly the same.

\section{References}

[1] Fainzilberg L. ECG Averaging Based on Hausdorff Metric. International Journal of Bioelectromagnetism, 2003, 5:236237.

[2] BIOPAC Systems. ECG Analysis using the Offline Averaging Mode. www.biopac.com

[3] Kim K, Lee Y H, Kwon H, et al. Averaging Algorithm Based on Data Statistics in Magnetocardiography. Neurology and Clinical Neurophysiology, 2004, 42: 357358.

[4] Braeklein M, Tchoudovski I, Moor C, Werthman M, Carlson S, Bolz A. Wireless Cardiological Monitoring of High-risk Patients at Home. Health Academy. 01/2004, 188-195.

[5] Egorouchkina K, Pang L, Tchoudovski I, Bolz A. Fast QRS detection algorithm for electrical cardioversion. ICMP and BMT 2005 to be published.

[6] Laguna P, Mark R G, Goldberger A and Moody G B. Database for Evaluation of Algorithms for Measurement of QT and Other Waveform Intervals in the ECG. Computers in Cardiology, 1997, 24: 673-676.

[7] Laguna P, Thakor N V, Caminal P, et al. New algorithm for QT interval analysis in 24-hour Holter ECG: performance and applications. Med. \& Biol. \& Comput., 1990, 28:67-73.

[8] The CSE working party. Recommendation for measurement standards in quantitave electrocardiographpy. European Heart Journal, 1985, 6:815-825.

Address for correspondence

Ksenia Egorouchkina

Corscience $\mathrm{GmbH} \& \mathrm{Co} \mathrm{KG}$

Henkestrasse 91, D-91052 Erlangen, Germany

e-mail: egorouchkina@corscience.de 